\title{
PROCESS AND SYSTEMS The Walton Centre neurology network - an equitable, sustainable and deliverable model for a large-scale neurology service
}

\author{
Authors: Nicholas A Fletcher, ${ }^{A}$ Martin Wilson, ${ }^{B}$ Julie Riley ${ }^{C}$ and Andrew Nicolson ${ }^{\mathrm{D}}$
}

\begin{abstract}
UK neurology has many different models of provision and a shortage of essential clinical staff. Services are sometimes unsatisfactory and there is much variation and inequality, especially in areas outside London where there are far fewer consultants. Some hospitals have much better staffed and resourced neurological services than others which may have far less provision or even no neurology service at all. There is no national strategy or agreed model of service delivery - local areas have evolved individual arrangements, often dictated by consultant availability. We describe, with clear operational details, a neurology network model in a large population, with outcomes. In many areas with limited resources it could, by re-organisation of current services, be considered instead of existing separate, variable and potentially inequitable local arrangements.
\end{abstract}

KEYWORDS: Neurology, service design, management, pathways

\section{Introduction}

Each year, about 650 per 100,000 people are diagnosed with a neurological disorder in the UK and prevalence in the population is about $6 \% .^{1-4}$ In $2015-16$ there were 474,401 neurological hospital admissions ${ }^{5}$ and in 2012-13 over 1 million outpatient appointments. $^{6}$

Unfortunately, the UK has too few neurologists. The Royal College of Physicians (RCP) reported 783 consultants in 2014 - or 1 per 83,000 people. ${ }^{7}$ In the EU and the USA there are 1 per 15,000 and 1 per 19,000 respectively. ${ }^{8}$ Moreover, British neurologists are extremely unevenly distributed. Using RCP data, ${ }^{7} \mathrm{NHS}$ data $^{9-11}$ and UK population figures, we estimate that about $25 \%$ of neurologists work in inner London where there is one consultant per 37,000 people compared with figures of between 1 per 71,000 and 1 per 106,000 in the rest of the country.

Authors: ${ }^{\mathrm{A}}$ consultant neurologist, Walton Centre NHS Foundation Trust, Liverpool, UK; ${ }^{B}$ consultant neurologist, Walton Centre NHS

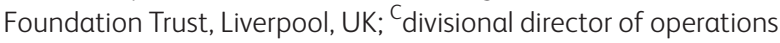
for neurology, Walton Centre NHS Foundation Trust, Liverpool, UK; ${ }^{D}$ consultant neurologist, Walton Centre NHS Foundation Trust, Liverpool, UK
This situation has unfortunate consequences. ${ }^{4,12}$ Some hospitals struggle to recruit and retain consultants and have minimal or no neurology service; ${ }^{12}$ others are closing their departments. ${ }^{13-17}$ Some with no service are less than 50 miles from large neurological units. ${ }^{13,16}$ In places, neurology centres in teaching hospitals offer variable support to nearby acute hospitals, ranging from consultants visiting several days a week to no formal support at all. Elsewhere, there are departments in acute (district) general hospitals (AGHs). These also vary, some are well resourced, others (sometimes nearby) are barely viable. ${ }^{12}$ The result is an inequitable patchwork of provision. ${ }^{4,12}$ The situation is compounded by local commissioning - an arrangement unlikely to deliver an equitable and sustainable service for a large population. ${ }^{2}$

Other difficulties have long been apparent. 2,4 Many doctors lack confidence with neurology ${ }^{18}$ - so called 'neurophobia' - despite neurology being within undergraduate training, the general practitioner (GP) curriculum and Membership of the Royal Colleges of Physicians examination. ${ }^{19}$ Outpatient services are often slow; coordination of care is poor. ${ }^{2,20}$ The management of even common problems like headache and seizures is inconsistent. ${ }^{21}$

The Association of British Neurologists (ABN) has written acute and elective care standards ${ }^{22}$ but they cannot be delivered everywhere at present. ${ }^{12,23}$ In 2011, the RCP and ABN recommended new 'local neurology' departments in AGHs. ${ }^{4}$ However, neuroscience centres were to remain alongside them but there was no clear guidance on how centres should deliver elective or acute neurology support to poorly staffed AGHs in the interim. The operational details of how such local neurology units would be resourced, the implications for centres, and the number of consultants required were unclear. Unfortunately, there is no likelihood of the necessary recruitment in many parts of the UK. Large-scale re-deployment of existing consultants is unlikely, would damage existing services and would still not suffice. The required infrastructure and support staff are unlikely to be available, nor the managerial expertise or the neurologists needed for on-call availability and necessary sub-specialty skills. Even in London, one suggested model for just acute neurology referrals in one hospital on weekdays required two-three consultants; ${ }^{24}$ elsewhere, such staffing levels may be possible in some units but are surely an impossible aspiration in others. As well as acute neurological care in acute general hospitals, about which the ABN and RCP were concerned, there are many more outpatients ${ }^{6}$ of whom an increasing number are complex and need sub-specialty expertise. 
Various reports have discussed these problems but not any realistic and deliverable solutions other than long-term aspirations to recruit many more consultants, 're-design' services in some unspecified way or list what they must offer. ${ }^{25,26}$ Neurology lacks any strategy that could be used until greater resources arrive in future.

In this paper, we describe - in clear operational terms - a realistic and feasible network model. Resources are deployed as equitably and effectively as possible in a large population of approximately 2.7 million.

\section{Population}

The service is based at The Walton Centre NHS Foundation Trust (WCFT) in Liverpool. Its mission is a regional service for Cheshire, Merseyside, south Lancashire and north Wales, a population of approximately 2.7 million people, in urban and rural environments (the 'core population'). Referrals regularly come from other areas populated by about 800,000 people, so there is a combined referral population of approximately 3.5 million. The population of Liverpool and adjacent clinical commissioning group (CCG) areas is 971,000 or $36 \%$ of the core population. ${ }^{27}$

\section{Organisation of the Service}

\section{WCFT}

The WCFT is based adjacent to University Hospital Aintree - an acute hospital with a major trauma centre and stroke unit. There are wards for neurology (29 beds) and neurosurgery (103 beds). There is a complex rehabilitation centre (10 acute rehabilitation beds, 30 additional level 1 beds), day ward, outpatient department and departments of neurosurgery, neurophysiology, neuroanaesthesia and intensive care ( 20 beds), neuroradiology, neuropsychology, pain medicine and neuropsychiatry. A brain injury unit provided by a mental health trust is co-located on the site and there is an adjacent oncology facility for stereotactic radiotherapy. WCFT is the only dedicated neurosciences trust in the UK. Its summary strategic plan is available. ${ }^{28}$

\section{Consultants}

There are 38 consultant neurologists; three are academic appointments, six work part time, one is the trust medical director, one a neurology clinical director, equating to 33.5 whole time equivalents. WCFT is responsible for recruitment, employment and management of all neurology consultants and provision of the service locally and for all linked partner hospitals. This equates to 1 consultant per 81,000 for the core population (below average for England outside London) and 1 per 105,000 for the whole population served.

> All consultants are employed by WCFT except for a university professor. All clinical governance, job planning, mandatory training and continuing professional development is at WCFT. All appraisals are at WCFT and the trust medical director is the responsible officer. All leave is managed centrally to maintain adequate service delivery at WCFT and linked hospitals.

$>$ All consultants offer general neurology and a sub-specialty and almost all work at WCFT and at a designated external linked hospital. Typically, job plans include 7.5 direct clinical care (DCC) and 2.5 supporting professional activity (SPA) sessions although there is flexibility to allow for variation in the mix of work. At WCFT, DCC sessions include clinics, administration ( 0.5 per clinic), ward work and on-call. There is a weekly consultant on call who is released from external activity to permit daily rounds on the neurology ward. Typically, neurologists are at an outside hospital on 2 days each week (except when on call). Commonly, two consultants 'share' a hospital, so there is a usually a 4-day on-site presence and one visits each week even if the other is on leave. $A$ linked hospital day comprises 2.5 DCC sessions - one for a clinic, one for in-patient consultations and a half for administration. A few other external clinics are provided (see below).

\section{Junior neurology staff}

There are foundation year 2 doctors, core medical trainees, specialist registrars and academic clinical fellows in neurology. All are based at WCFT but all middle grade neurology doctors have periods of time at a linked hospital on 1 or more days per week. The organisation of the training programmes for these doctors is outside the scope of this article.

\section{Locations of WCFT consultant neurologist service}

Outpatient clinics and inpatient referrals are at WCFT and at 12 additional external 'linked hospital' or 'satellite' locations (Fig 1). Almost all locations have a WCFT neurologist on-site for 4 days each week. There will be a general neurology clinic and a ward liaison session on each of these days. On the Isle of Man, two WCFT consultants alternately provide weekly overnight visits with clinics and ward consultations over 2 days.

> On other days, medical staff at these locations can telephone the WCFT on-call team or one of their visiting consultants for advice, to request transfer of a patient or - currently being developed at two pilot sites only - a telemedicine consultation (see future developments below). It may be decided (after WCFT advice if required) to await the next visit if clinically appropriate.

$>$ Stroke ward sessions are provided each week at two outside hospitals by a sub-specialty cerebrovascular neurologist, in addition to the other ward consultation sessions at those hospitals.

$>$ Consultants also provide some clinics at non-acute sites (with no emergency department). These are at two other hospitals and a primary care centre. There is also a monthly clinic at the Liverpool Women's Hospital for pregnant women with neurological conditions and two clinics each month in Oswestry. There are weekly multiple sclerosis (MS)

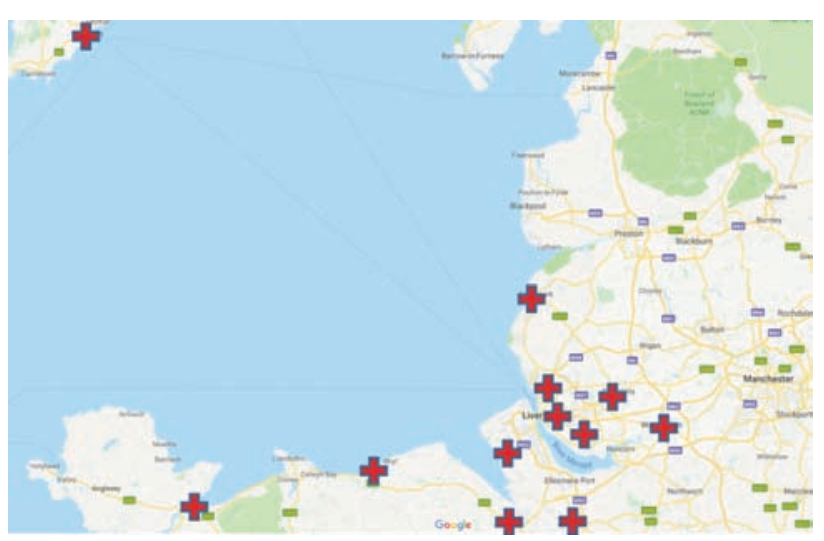

Fig 1. Locations of acute ward consultation sites (linked acute sites). 


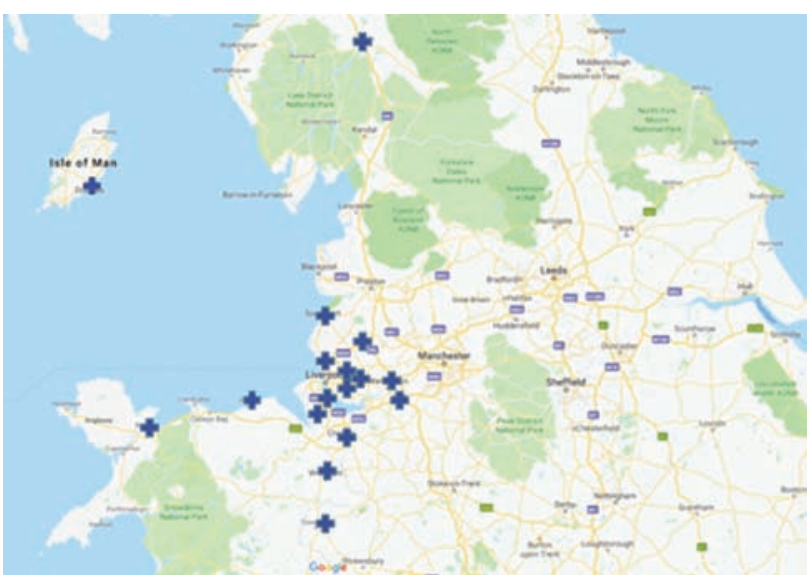

Fig 2. Location of all The Walton Centre NHS Foundation Trust provided outpatient clinic sites.

clinics in north Wales and Chester, a behavioural neurology/ cognitive disorders clinic twice a month in north Wales and

WCFT has provided a quarterly movement disorders clinic at Penrith hospital. All outpatient clinic locations are shown in Fig 2.

> At WCFT, there are general neurology (secondary care) clinics for the local population (and those from other areas who choose a WCFT appointment). There is also a comprehensive range of tertiary sub-specialty clinics (including transitional clinics with input from Alder Hey Children's Hospital consultants) and a weekly emergency clinic.

\section{Outpatients}

For outpatients seen at WCFT, all investigations are undertaken there. Externally, routine blood tests and radiology are ordered at the satellite hospital, as are most computed tomography and magnetic resonance scans. Neurophysiology can be carried out by WCFT neurophysiologists at Royal Liverpool Hospital but otherwise is done at WCFT. Complex neuroradiology is undertaken at WCFT. If necessary, satellite hospital scans are readily reviewed at WCFT via the regional radiology image transfer system or by image transfer and at weekly neuroradiology conferences. Elective lumbar puncture, nerve or muscle biopsies are done at WCFT, usually as day cases.

\section{Inpatients}

Inpatients are admitted centrally to WCFT for specialist investigations or treatment. These include elective admissions from WCFT or satellite clinics as well as urgent patients transferred from any hospital in the network. Patients seen as ward referrals in linked hospitals are, whenever possible, managed by the local medical team with a neurological management plan and, if required, subsequent on-site review, follow-up telephone advice to the medical team or neurology elective care after discharge. Patients requiring investigations or treatment that cannot be done locally or frequent neurology review are transferred to WCFT. Patients awaiting transfer are admitted on the basis of clinical urgency and with input from the consultant on call.

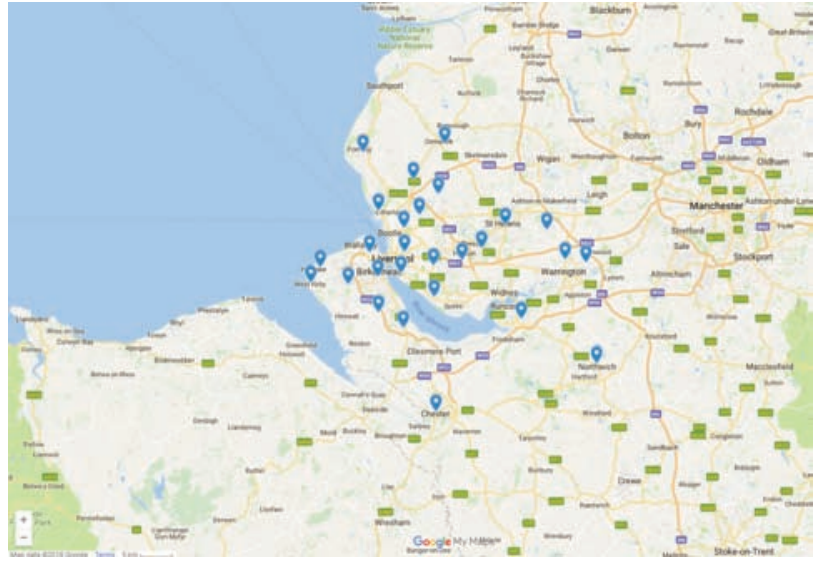

Fig 3. Location of nurse clinic sites (integrated neurology nurses).

\section{Specialist nurses}

There are two groups of specialist nurses; all are employed and managed by WCFT. There are condition specific nurses in MS, epilepsy, Parkinson's and movement disorders, neuromuscular disease, motor neurone disease and neuromyelitis optica. They provide (disease specific) outpatient and telephone clinics mainly at WCFT and some external sites. There are also seven integrated neurology nurses (INNs) who have undergone formal training in neurological disorders. INNs are community based and provide 34 clinics per week at 27 community locations in the English part of the WCFT region plus 13 telephone clinics each week as well as (where necessary) domiciliary and care/nursing home visits. INNs also visit seven local English emergency departments to advise on pathway utilisation, neurological management and identification and to offer clinical review of frequent neurological attenders. Training (for example emergency management of seizures) is also provided in the community. INNs liaise with other agencies regarding social care, appliances, physiotherapy, occupational therapy, speech and language therapy, continence services or district nursing. They can also quickly obtain advice from the patient's WCFT consultant if required. INN clinic locations are shown in Fig 3.

\section{Consultant and nurse telephone advice lines}

A consultant telephone advice line (CALs) for GPs operates for 2 hours each day (Monday-Friday). GPs can call the on-call team at other times. The consultant on call carries a designated mobile phone to receive CAL calls. The call outcomes are recorded, audited and reviewed. Nurse telephone advice lines (NALs) operate in the MS, epilepsy, Parkinson's and movement disorders, and neuromuscular teams. To avoid having to leave messages on answering machines (and a backlog of calls developing), patients initially speak with an administrator who notes the nature of the call and allocates a 'call-back' by an appropriate nurse (disease specific or INN) at a designated time. This also avoids the patient having to stay by the phone awaiting a return call. NAL calls are also audited.

\section{Referrals, triage and centralised management of appointments and waiting times}

All referrals, whether received at WCFT or satellite hospital offices are handled by a centralised Patient Access Centre (PAC) at 
WCFT. Referrals are triaged by consultants and allocated to the most appropriate WCFT or satellite clinic. Patients are offered appointments at their preferred location whenever possible; if not, at the most acceptable and appropriate alternative. PAC has access to all clinics and sites, allowing flexibility to deal with demand and optimal use of all available capacity. Appointments becoming available in clinics (particularly sub-specialty) because of cancellations or fluctuation in demand for a particular clinic can be used for other appropriate referrals. The centralised handling of referrals and appointments allows monitoring of demand, activity, triage and waiting times. Acute ward referrals are sent to the visiting neurologists' office at that hospital by its usual internal referral systems. They are triaged and seen by the next visiting consultant. All are logged as WCFT activity.

\section{Secretarial support at linked sites}

Neurology secretarial support is provided at each acute site as a point of contact for referrals and clinical administration. The secretary can also provide most information about the service needed by local clinical staff.

\section{Activity and outcomes}

\section{Outpatients}

In 2017-2018 WCFT neurology saw 71,659 outpatients - 23,007 new patients and 48,652 reviews. Approximately $30 \%$ of new referrals were seen at a linked hospital, a proportion which has varied between 41 and $29 \%$ in recent years. Referral to treatment time (RTT) performance at March 2018 was $97 \%$ of open pathways less than 18 weeks in NHS England (target $92 \%$ ) and $96 \%$ of open pathways less than 26 weeks for NHS Wales (target 95\%).

\section{Inpatients at WCFT}

533 neurology patients were admitted in 2017-2018 (not including patients admitted to neurosurgery directly) - 275 were elective (52\%) and $258(48 \%)$ were emergencies. The proportion of emergency admissions from the Liverpool and adjacent CCG areas was $38 \%$, which is consistent with the proportion of the WCFT core population in those areas (36\%). Emergencies were therefore as likely to be admitted from a remote linked hospital as a more local one.

\section{Inpatients seen at linked hospitals}

In 2017-2018, WCFT neurologists saw 4,794 inpatients at linked hospitals (excluding the Isle of Man) - 2,341 (49\%) did not require any subsequent review, $2,152(45 \%)$ were discharged with outpatient review, only $211(4 \%)$ required subsequent on-site review and $90(2 \%)$ required transfer to WCFT. An additional 70 patients were transferred as emergencies to WCFT from linked hospitals after telephone referral. All other emergency admissions were admitted directly from home or an outpatient clinic.

\section{Consultant advice line}

From March 2017 to March 2018, there were 839 calls to CALs from GPs. The nature of these is shown in Fig 4 and outcomes in Fig 5. Headaches, isolated sensory or motor symptoms or seizures were the main problems referred; $76 \%$ concerned

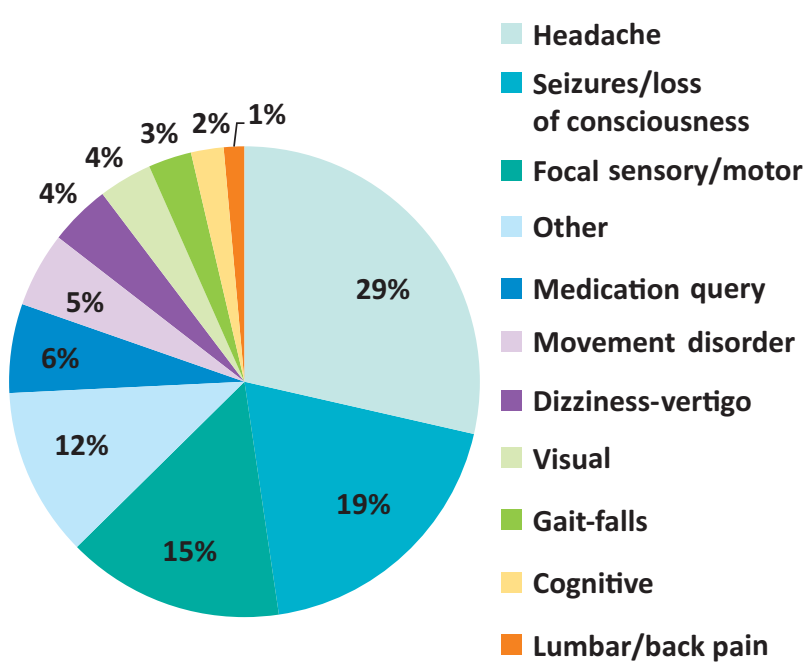

Fig 4. Clinical nature of calls to consultant telephone advice lines (March 2017 to March 2018).

patients with new neurological problems. Many (57\%) of these calls required advice only; only $2 \%$ required local admission and $35 \%$ required an outpatient appointment. An online survey of 20 GPs was conducted after using the service; 17 who responded scored it a mean of 9.2 on a scale of $0-10$ for usefulness and all responders would use the service again. Qualitative feedback was overwhelmingly positive.

\section{Recent service developments}

In 2016, WCFT was chosen as a NHS England acute care vanguard site. This was an opportunity to enhance the service in partnership with WCFT's seven linked English acute hospitals. The elements of the project were as follows.

\section{> Development of a seizure management pathway in} emergency departments and acute medical units of linked hospitals. ${ }^{29}$ This was in response to the results of the National Audit of Seizure management in Hospitals revealing considerable variation in management and referral of patients after seizures. ${ }^{21}$ It has been established in seven partner acute trusts. Its elements are:

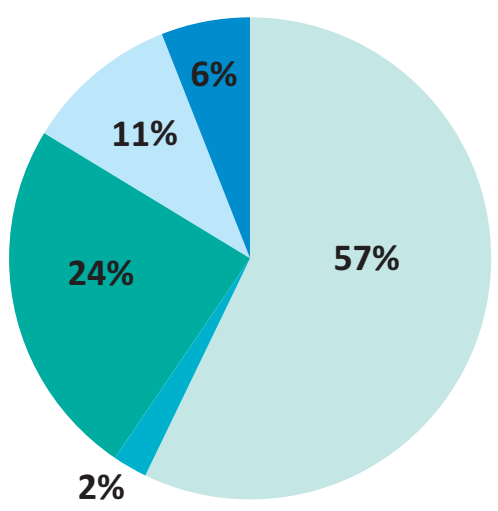

Advice only

Admit locally as emergency

Refer to routine outpatient department

\section{Refer to emergency clinic}

Unknown

Fig 5. Outcome of calls to consultant telephone advice lines (March 2017 to March 2018). 
> clear admission and discharge criteria to avoid unnecessary admission

> guidance notes on management of patients requiring admission, on status epilepticus, and about obtaining inpatient advice or consultation if needed

$>$ a simple pathway for all seizure patients to be referred quickly to WCFT after discharge from the emergency department or an acute medical ward

$>$ to reduce repeat emergency department attendances and re-admissions

> patient information for all patients discharged from emergency department or inpatient care after seizures.

The outcome has been analysed between 2016 and 2018. More seizure patients have been referred $(+23 \%)$; the proportion of patients seen in a neurology clinic after a seizure related admission has increased from 34 to $42 \%$ and the proportion of seizure patients admitted to local linked hospitals who are not under follow-up by the neurology service has fallen from 58 to $48 \%$. However, the referral rate after admission of new (or not under review) seizure patients remains too low at $21 \%$. There has been no effect yet on re-admission or emergency department re-attendance rates of patients after seizure admissions. Further analysis and discussion of these results will be the subject of a separate publication.

> A primary care headache pathway. ${ }^{29}$ The pathway was to reduce avoidable referrals to secondary care and increase identification and referral of serious headache disorders. This has been adopted by seven CCGs after extensive engagement and GP educational sessions. The effect of this initiative between 2016 and 2018 has been a $24 \%$ reduction in headache referrals to WCFT, the number of new patients seen in neurology outpatients with headache has reduced by $22 \%$, attendances of patients diagnosed with migraine have reduced by $20 \%$ and the number of headache patients discharged after one attendance (a surrogate marker for patients who could be managed in primary care) has fallen by $42 \%$. These results indicate that more patients with migraine and other primary headache disorders have been offered treatment in primary care rather than hospital referral.

$>$ A secondary care acute headache pathway ${ }^{29}$ to assist emergency department and acute medical staff. The aim is to improve identification and management of serious acute headache disorders and avoid unnecessary admission (and referral) of patients with primary headache disorders such as migraine. This project is currently being introduced so no outcomes are available.

> To expand the CAL to $\mathbf{2}$ hours each day. Also improving recording and documentation of the advice line consultations. The results are noted in activity and outcomes above.

$>$ An increase of the INN concept (see specialist nurses above) to cover seven CCG areas. Competency based training was developed and formalised.

> Centralised handling of NAL calls to provide more efficient call-back arrangements and avoid delays and use of voicemail messages. Patient feedback has been very positive.

> Development of a telemedicine facility to offer remote consultation with a WCFT consultant neurologist for acute medical unit staff. This has been tried at two pilot sites with plans to expand the service to other linked hospitals in time.

$>$ Development of a functional neurology service at WCFT to assist in the management of patients with medically unexplained symptoms.

\section{Contracts and partnerships}

WCFT has contracts with seven English CCGs, Betsi Cadwaladr University Health Board (BCUHB; north Wales), NHS England specialist commissioners and the Isle of Man. With the exception of the Isle of Man (which has different contractual arrangements), all outpatients seen at linked hospitals are WCFT activity; WCFT retains responsibility for (and manages) RTT pathways at linked sites. Although ward consultations by WCFT clinicians at linked hospitals are also WCFT activity, these inpatients remain officially under the care of local clinicians. Contracts between WCFT and commissioners cover activity at WCFT, local satellite clinics and linked hospital and community sites.

The use of facilities, secretarial and diagnostic services at linked partner hospitals is managed by service level agreements between WCFT and the relevant English acute trusts and BCUHB.

\section{The future}

Following the NHS England acute care vanguard initiative, we aim to offer the same clinical pathways in north Wales; Welsh GPs, patients and acute sites can already use the CAL and NAL. It is intended to increase all sites to 4 days of consultant visits per week. Telemedicine pilots at two sites will hopefully, in time, be followed by this service at all linked sites and this will probably require a separate on-call consultant rota at WCFT. There is likely to be further development and use of electronic referrals, especially with the seizure pathway and the development of an electronic GP headache referral system linked to the headache pathway.

\section{Discussion}

The problems of UK neurology have often been described, but deliverable solutions have not. A focus on proposals that are currently unrealistic $^{4,8,22}$ or unlikely outside London ${ }^{24,30}$ or other well-staffed localities should not hold back wider development of less utopian but operationally feasible improvements.

The model we have described is not perfect. A fully resourced neurology service in every acute hospital would, in some respects, be preferable. It would certainly offer rapid neurological management of acute admissions. ${ }^{4,24}$ Unfortunately however, we see several problems. At present, something approaching such a service is available in some acute hospitals ${ }^{24}$ but not in others, and sometimes near a hospital with minimal or no service at all. ${ }^{13-16}$ Highly inequitable variation is not unexpected if scarce, unequally distributed staff are sought by multiple locally commissioned services. The staff and financial resources to provide a 'local neurology' model in all NHS acute hospitals are unlikely in the foreseeable future - hence UK neurology's persisting difficulties after decades of arguments for this approach. At present, "local neurology' is not, and will not be, deliverable across the UK.

In addition, locally delivered services would be entirely unable to deliver the full range of subspecialty services, so 'tertiary' neurology centres would still be required.

By contrast, the Walton network model is a realistic, deliverable option. It avoids the problem of a neurologically well-provided hospital near another with inadequate provision. In short, 4 days a week everywhere (with formal support arrangements on other days) may be better (and is certainly more equitable) than 5 or even 7 days in some hospitals and little or nothing in others.

A key argument for the 'local neurology' approach has been deployment of neurologists in AGHs to see inpatients more 
quickly, even if this reduces outpatient care. ${ }^{4,12}$ In our experience, of almost 5,000 such patients seen in a year by our consultants, only $2 \%$ required transfer to WCFT, $4 \%$ needed another inpatient review and only $45 \%$ required outpatient follow-up. Elsewhere, $25 \%$ of acute referrals did not need admission. ${ }^{24}$ Although we do not dispute the value of timely assessment of such patients and that a few have serious neurological problems, it is questionable to base our whole neurology strategy ${ }^{4}$ on the needs of this patient group; outpatients also have serious neurological problems and can become inpatients if not seen quickly.

A network model can reduce clinical variation, especially if combined with clinical pathways to be used in primary and secondary care. It also offers consistent clinical governance, job planning, appraisal and continuing medical education. Research and audit can be done on a larger scale and results of the latter communicated to a wider service than is possible with a multitude of separate local services. Prospects for recruitment are also likely to be better.

This description of a network model offers an option, in clearly described operational terms, for clinicians and managers who are willing and able to reorganise and integrate existing neurological services to offer a more equitable and consistent approach to larger populations - rather than continue with highly variable local provision.

\section{Conflicts of interest}

Dr Nicolson is the medical director of The Walton Centre NHS Foundation Trust.

\section{References}

1 MacDonald BK, Cockerell OC, Sander JW, Shorvon SD. The incidence and lifetime prevalence of neurological disorders in a prospective community-based study in the UK. Brain 2000;123:665-76.

2 House of Commons Committee of Public Accounts. Services to people with neurological conditions: progress review. London: The Stationery Office, 2016.

3 The Neurological Alliance. Neuro numbers. London: The Neurological Alliance, 2014.

4 Royal College of Physicians, Association of British Neurologists. Local adult neurology services for the next decade. Report of a working party. London: RCP, 2011.

5 Neurology Intelligence Network, Public Health England. Neurology Intelligence Network hospital activity compendium. Public Health England, 2017.

6 National Mental Health, Dementia and Neurology Intelligence Network. Neurology outpatient analysis: National Neurology Intelligence Network data briefing. Public Health England, 2015.

7 Federation of the Royal Colleges of Physicians of the UK. Census of consultant physicians and higher specialty trainees in the UK 2014-15: data and commentary. London: RCP, 2016.

8 Morrish PK. Inadequate neurology services undermine patient care in the UK. BMJ 2015:350:h3284.

9 NHS Digital. NHS Workforce Statistics, December 2017. NHS Digital, 2018.

10 NHS Digital. NHS occupation codes. NHS Digital, 2018 https:// digital.nhs.uk/data-and-information/areas-of-interest/workforce/ nhs-occupation-codes.

11 ISD Scotland, NHS National Services Scotland. NHS employed staff working in hospital, community and public health services (HCHS). Edinburgh: ISD Scotland, 2018. www.isdscotland.org/Health-Topics/ Workforce/Publications/2018-06-05/Medical-and-Dental.asp.

12 Association of British Neurologists. ABN Acute Neurology report. London: ABN, 2017. www.theabn.org/news/abn-acute-neurology-report.html.
13 BBC News. Shrewsbury and Telford hospital services close to new patients. BBC, 2017. www.bbc.co.uk/news/uk-englandshropshire-39231208.

14 The Shrewsbury and Telford Hospital NHS Trust. Temporary suspension of Neurology services to new referrals to be extended. The Shrewsbury and Telford Hospital NHS Trust, 2018. www.sath.nhs.uk/news/ temporary-suspension-neurology-services-new-referrals-extended.

15 Pidluznyj S. Doctors' shortage crisis worsens: Lincolnshire neurology service closed to new patients. Lincoln: Lincolnshire Reporter, 2016.

16 Burton Hospitals NHS Foundation Trust. Statement on Neurology Services at Burton Hospitals NHS Foundation Trust. Burton Hospitals NHS Foundation Trust, 2017.

17 The Northern Echo. MSPs told of neurology 'recruitment crisis'. The Northern Echo, 2018.

18 Pakpoor J, Handel AE, Disanto G et al. National survey of UK medical students on the perception of neurology. BMC Medical Education 2014;14:225.

19 Membership of the Royal Colleges of Physicians of the United Kingdom. MRCP(UK) examinations. London: MRCP(UK), 2018. www.mrcpuk.org/mrcpuk-examinations.

20 The Neurological Alliance. The invisible patients: Revealing the state of neurology services. London: The Neurological Alliance, 2015.

21 Dixon PA, Kirkham J], Marson AG et al. National Audit of Seizure management in Hospitals (NASH): results of the national audit of adult epilepsy in the UK. BMJ Open 2015;5:e007325.

22 Association of British Neurologists. ABN guidelines for unscheduled care and non-urgent neurological conditions. London: ABN, 2014. www.theabn.org/resources/abn/g/abn-guide-unscheduled-care.html.

23 The Neurological Alliance, Vibert S. NICE letter. Watford: The Neurological Alliance, 2018. www.neural.org.uk/assets/pdfs/201801-nice-letter-2018.pdf.

24 Moodley KK, Nitkunan A, Pereira AC. Acute neurology: a suggested approach. Clin Med 2018:18:418-21.

25 NHS Thames Valley Strategic Clinical Network. Transforming community neurology. What commissioners need to know. Part A Transformation guide. NHS, 2016. http://tvscn.nhs.uk/wp-content/ uploads/2016/06/Transforming-Community-Neurology-Part-ATransformation-Guide-version-1.pdf.

26 Association of British Neurologists. Neurology Commissioning Toolkit (NCT). London: ABN, 2016. www.theabn.org/media/ Documents/ABN\% 20publications/ABN\% 20Neurology \% 20 Commissioning \% 20Toolkit\% 202016.pdf.

27 Office for National Statistics. Small area population estimates, England and Wales: mid-2016. ONS, 2017. www.ons.gov.uk/ peoplepopulationandcommunity/populationandmigration/ populationestimates/bulletins/annualsmallareapopulationestimates/ mid2016.

28 The Walton Centre NHS Foundation Trust. Summary strategic plan 2014-19. The Walton Centre NHS Foundation Trust, 2014. https:// assets.publishing.service.gov.uk/government/uploads/system/ uploads/attachment_data/file/390328/WALTON_Publishable_ Summary_Strategic_Plan_1415.pdf.

29 The Walton Centre NHS Foundation Trust. Pathways and downloads for health professionals. The Walton Centre NHS Foundation Trust. www.thewaltoncentre.nhs.uk/473/pathways-for-healthprofessionals.html.

30 NHS London Clinical Networks. Hyper acute neurology services. NHS, 2016. www.londonscn.nhs.uk/wp-content/uploads/2016/12/ neuro-acute-hans-122016.pdf.

Address for correspondence: Dr Nicholas A Fletcher, consultant neurologist, Walton Centre NHS Foundation Trust, Liverpool L9 7LJ, UK.

Email: nick.fletcher2@thewaltoncentre.nhs.uk 